\title{
PRIMER REGISTRO FÓSIL DEL GÉNERO Dicyphus (INSECTA: HEMIPTERA: MIRIDAE); MIOCENO INFERIOR DE TERUEL (ESPAÑ)
}

\author{
Enrique PEÑALVER' y Manuel BAENA ${ }^{2}$ \\ ' Dpto. Geología, Fac. Biología, Universitat de València, C/Dr. Moliner 50, E-46100 Burjassot, \\ Valencia (España).E-mail: penalver@uv.es \\ 2 Dpto. de Biología y Geología, I.E.S. "Antonio Gala”, Avda. de la Paz, s/n. apartado de correos n \\ 14, E-14700 Palma del Río, Córdoba (España).E-mail: jsusin@chopo.pntic.mec.es
}

Peñalver, E. y Baena, M. 2000. Primer registro fósil del género Dicyphus (Insecta: Hemiptera: Miridae); Mioceno Inferior de Teruel (España). [The first fossil record of the genus Dicyphus (Insecta: Hemiptera: Miridae); Lower Miocene of Teruel (Spain).] Revista Española de Paleontología, 15(1), 49-56. ISSN 0213-6937.

\begin{abstract}
Dicyphus rubusensis nov. sp (Insecta: Hemiptera: Miridae) is described from four specimens from Lower Miocene (Lower Aragonian) deposits of Rubielos de Mora (province of Teruel) in eastern Spain. It is the first fossil record of the genus Dicyphus. From a morphometric characterization of this new species, we conclude that it is a member of the hyalinipennis group. The principal difference between this species and other representatives of the hyalinipennis group is the absence in the former of tibial spines longer than the tibial diameter of meso and metatibiae. These biometric features are similar to extant Dicyphus cerastii Wagner, 1951, but the fossil species is smaller and bears different somatic proportions. This species probably possessed a taxonomically broad spectrum of consumed plant host although its host had a pilose and/or glandular surface.
\end{abstract}

Keywords: Insecta, Hemiptera, Miridae, Dicyphus, nov. sp., Lower Miocene, Spain.

\section{RESUMEN}

Se describe Dicyphus rubusensis nov. sp. (Insecta: Hemiptera: Miridae) a partir de cuatro ejemplares hallados en ritmitas bituminosas del Mioceno Inferior (Aragoniense Inferior) de Rubielos de Mora (provincia de Teruel), en el este de España. Este es el primer registro fósil conocido del género Dicyphus. La nueva especie se puede incluir en el grupo hyalinipennis, separándose de todas las especies que lo integran por la ausencia de setas espinosas de mayor longitud que el diámetro tibial en meso y metatibias. En el seno del grupo, por su biometría, se parece a la especie actual Dicyphus cerastii Wagner, 1951, pero se diferencia por su menor tamaño y por las distintas proporciones somáticas. Esta especie probablemente poseía un amplio espectro taxonómico de plantas hospedadoras de las que se alimentaría, si bien éstas poseerían superficies pilosas y/o glandulares.

Palabras clave: Insecta, Hemiptera, Miridae, Dicyphus, nov. sp., Mioceno Inferior, España.

\section{INTRODUCCIÓN}

Los míridos constituyen la familia de heterópteros más extensa y diversa, con cerca de 10.000 especies actuales (Schuh, 1995). Se reconocen fácilmente por su delicadeza, su tamaño generalmente pequeño, la ausencia de ocelos, un rostro de 4 segmentos, un cuneus generalmente presente y un embolium indiferenciado. Actualmente la mayoría de ellos se alimentan de jugos vegetales, pero otros son depredadores de artrópodos. A pesar de su escasa esclerotización, es un grupo relativamente abundante en el registro fósil de Hemiptera y la mayor parte de los fósiles de míridos se han encontrado en sedimentos laminados lacustres del Oligoceno de Colorado (U.S.A.) y en el ámbar del
Eoceno/Oligoceno del Báltico (Carpenter, 1992). Los restos más antiguos conocidos pertenecientes a esta familia corresponden al Kimmeridgiense (Jurásico Superior) de Rusia (Labandeira, 1994). Labandeira seguramente hace referencia al estudio realizado por Popov (1968) de los heterópteros fósiles de Karatau (Kazakhstan) en el que describe el género Scutellifer y la especie S. karatavicus, dentro de la nueva tribu Scutelliferini.

Las citas paleontológicas de Miridae en España se limitan al hallazgo de dos ejemplares en el Mioceno. Un ejemplar fue estudiado por Gil Collado (1926), que lo asignó entonces a la familia Capsidae, actual Miridae, y provenía del yacimiento de "La Rinconada" del Mioceno Inferior de la cuenca de Ribesalbes-Alcora (Castellón). 


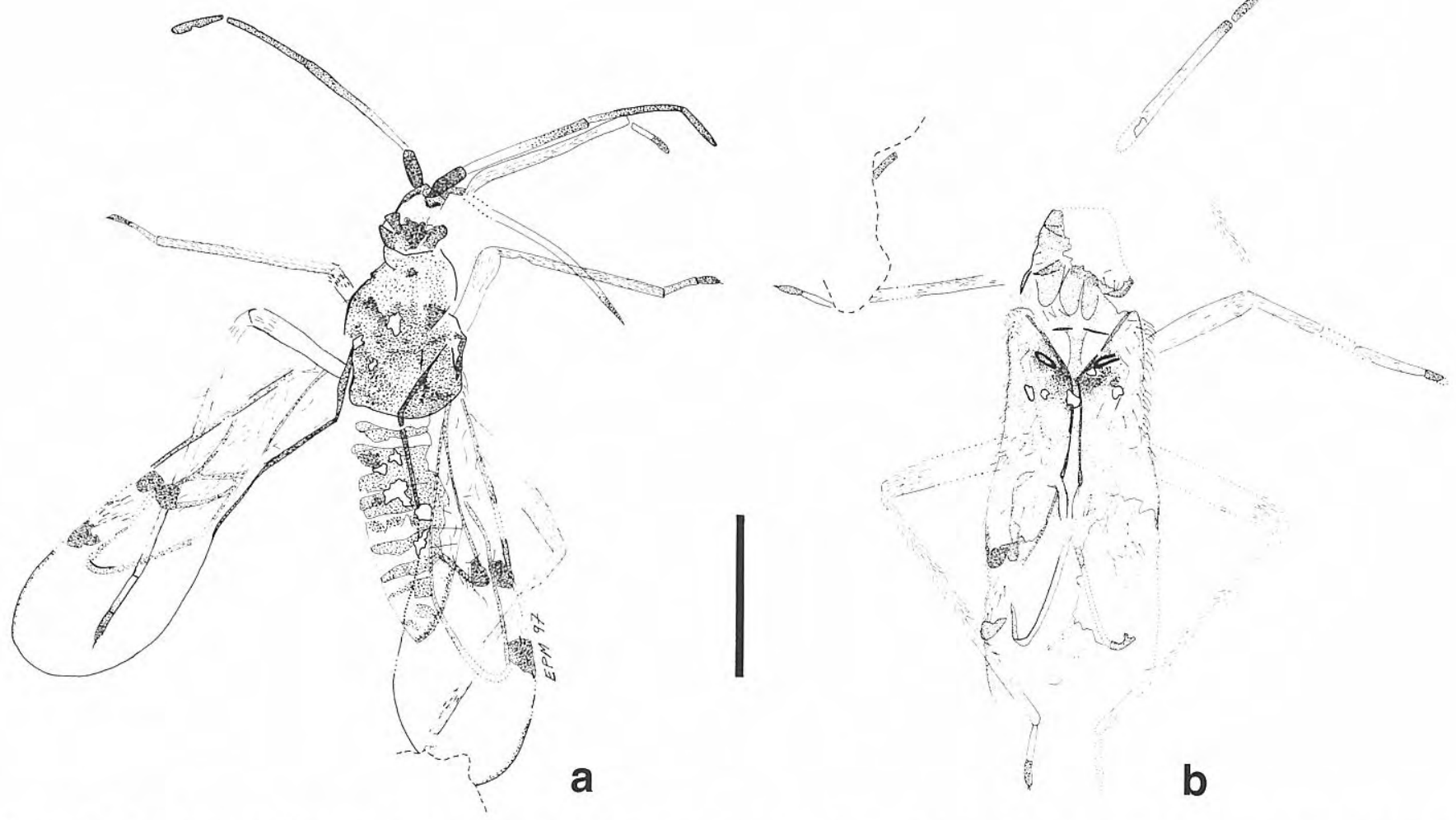

Figura 1. Ejemplares de Dicyphus rubusensis nov. sp. (Hemiptera: Miridae) de Rubielos de Mora. a) ejemplar MPV-566-RM, holotipo. b) ejemplar MPV-2244-RM, paratipo. Dibujos a cámara clara. Escala $1 \mathrm{~mm}$.

Desafortunadamente, la descripción es poco detallada y el ejemplar, originalmente depositado en las colecciones del Museo Nacional de Ciencias Naturales, está perdido en la actualidad. La segunda cita corresponde a un Miridae indet. (MPV-60-RM), hallado en el yacimiento de Rubielos de Mora en Teruel (Martínez-Delclòs et al., 1991), probablemente perteneciente al género Phytocoris Fallén.

El material estudiado en este artículo incluye el ejemplar MPV-70-RM, que fue clasificado por MartínezDelclòs et al. (1991; Lám. I, fig. 4) como un Stenocephalidae, posiblemente del género Dicranocephalus. La revisión de este espécimen ha puesto de manifiesto que corresponde al género Dicyphus. Otros tres especímenes completan el material estudiado: dos detectados en la colección del Museo de Ciencias Naturales de Valencia y otro del Museo de Paleontología de la Universidad de Zaragoza.

\section{AFLORAMIENTOS DE RUBIELOS DE MORA}

Los afloramientos con insectos fósiles de la cuenca de Rubielos de Mora (Teruel) están constituidos por ritmitas bituminosas lacustres originadas en el fondo de un gran lago meromíctico (Martínez-Delclòs et al., 1991; Montoya et al., 1996) durante el Aragoniense Inferior (Mioceno Inferior; 19-18 millones de años aprox.) Esta cuenca, limitada por fallas normales, se localiza en el sector sureste de la Cordillera Ibérica (Anadón et al., 1989). El afloramiento de "Río Rubielos", del cual provienen los especímenes estudiados, es uno de los más ricos en insectos fósiles de la cuenca junto al de "El Porpol". En todos los afloramientos destaca la buena conservación de los caracteres más delicados de los insectos, como la venación de las alas, patrones de coloración, etc. Así, la presencia de organismos de "cuerpo blando" y la detallada conservación de los mismos hacen incluir estos afloramientos entre los del tipo Konservat-Lagerstätte, o de conservación excepcional, los cuales son muy poco comunes incluso a nivel mundial (Martínez-Delclòs et al., 1991; Peñalver y Seilacher, 1995).

\section{SISTEMÁTICA}

ORDEN HEMIPTERA Linnaeus, 1758

SUBORDEN HETEROPTERA Latreille, 1810

INFRAORDEN CIMICOMORPHA Leston, Pendergrast and Southwood, 1954

Superfamilia MIROIDEA Hahn, 1833

Familia Miridae Hahn, 1833

Subfamilia Bryocorinae Baerensprung, 1860

Tribu Dicyphina Reuter, 1883

Género Dicyphus Fieber, 1858

Especie tipo: Capsus collaris Fallén, $1807=$ Dicyphus errans (Wolff, 1804). Actual. 


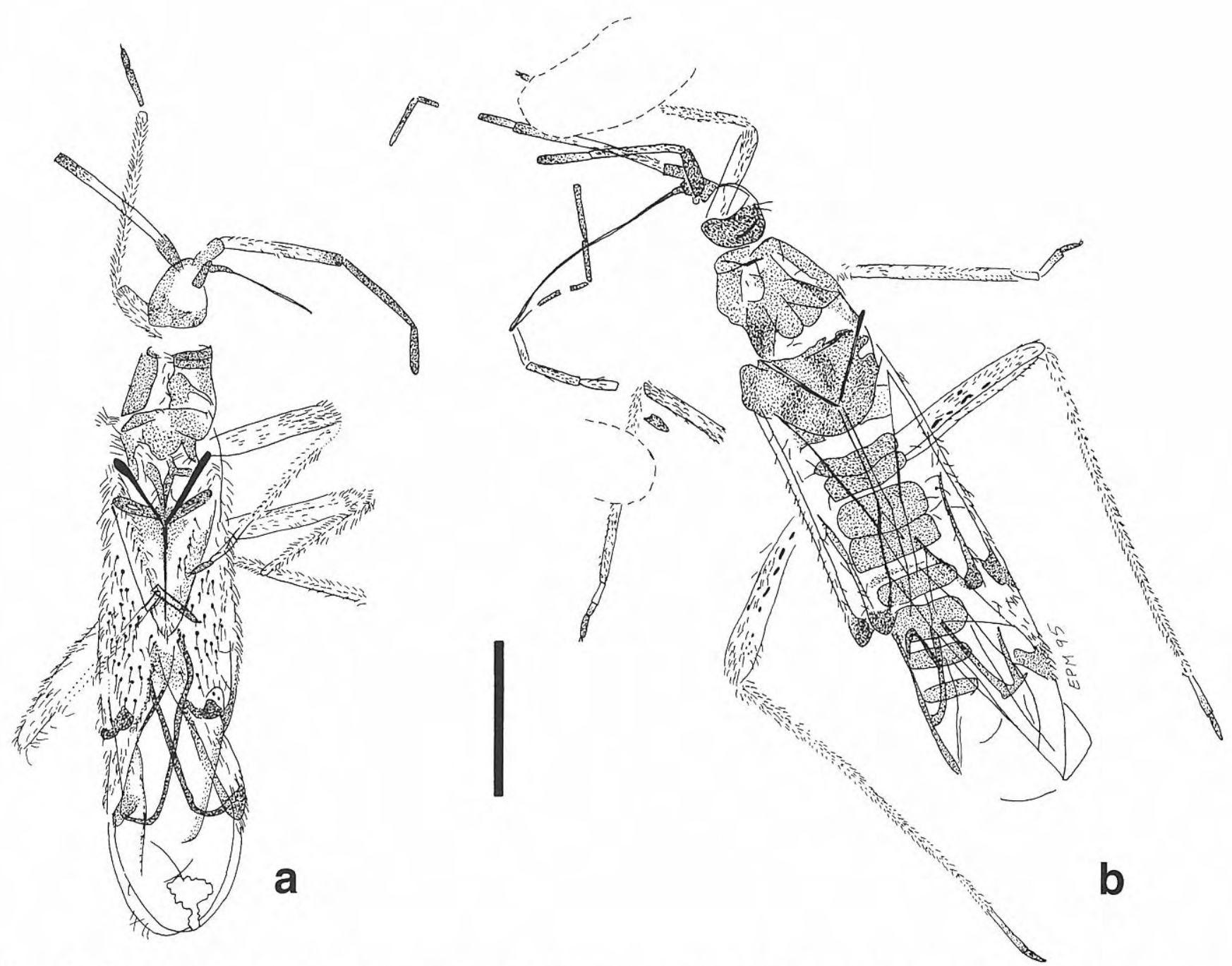

Figura 2. Ejemplares de Dicyphus rubusensis nov. sp. (Hemiptera: Miridae) de Rubielos de Mora. a) ejemplar MPV-70-RM, paratipo. b) ejemplar MPZ 97/555 paratipo. Dibujos a cámara clara. Escala $1 \mathrm{~mm}$.

Dicyphus rubusensis nov. sp.

Figs. 1-3; Lám. I

1991 Estenocefálido, posiblemente Dicranocephalus; Martínez-Delclòs, Peñalver y Belinchón, Lám. I; fig. 4.

Holotipo: Ejemplar MPV-566-RM (Fig. 1.a y Lám. I, figs. 1 y 3). Adulto y macróptero. Este espécimen fue hallado en el afloramiento denominado "Río Rubielos" (ver Montoya et al., 1996), concretamente en RR3. Colección del Museo de Ciencias Naturales de Valencia (antes Museo Paleontológico de Valencia).

Paratipos: Ejemplares MPV-70-RM (Figs. 2.a, 3 y Lám. I, fig. 4) y MPV-2244-RM (Fig. 1.b), hallados en el afloramiento denominado "Río Rubielos" (ver Montoya et al., 1996), concretamente en RR3, y pertenecientes a las colecciones del Museo de Ciencias Naturales de Valencia (antes Museo Paleontológico de Valencia), y MPZ 97/555 (Fig. 2.b y Lám. I, figs. 2, 5 y 6), hallado en RR2, y perteneciente a la colección del Museo de Paleontología de la Universidad de Zaragoza. Adultos y macrópteros.
Stratum typicum: Ejemplares hallados en ritmitas bituminosas de origen lacustre del Aragoniense Inferior (Mioceno Inferior) de la cuenca de Rubielos de Mora (Cadena Ibérica).

Locus typicus: Afloramiento "Río Rubielos" del yacimiento de Rubielos de Mora, provincia de Teruel, España (ver Montoya et al., 1996).

Derivatio nominis: rubusensis hace referencia a la localidad donde se encuentra el yacimiento del cual proceden los ejemplares ("Rubielos" deriva del latín rubus).

Diagnosis: La nueva especie se puede incluir en el grupo hyalinipennis, separándose de todas las especies que lo integran por la ausencia de setas espinosas de mayor longitud que el diámetro tibial en meso y metatibias.

Due to his morphometric characteristics, this new species may be included in the hyalinipennis group; the main difference between this new species and other representatives of the hyalinipennis group is the absence in the former one of tibial spines longer than the tibial diameter of meso and metatibiae. 


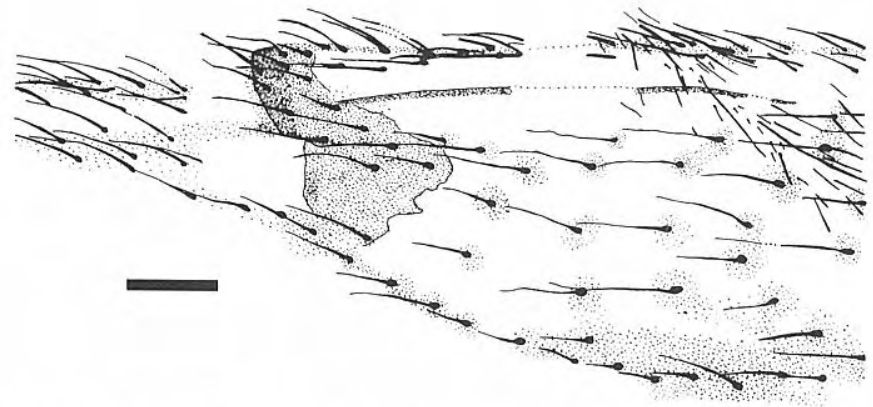

Figura 3. Detalle de la coria del paratipo MPV-70-RM de Dicyphus rubusensis nov. sp. (Hemiptera: Miridae) en el que se aprecian dos manchas, las setas de la membrana con inserciones oscuras y las setas semierectas del borde costal. Dibujo realizado a partir de una fotografía al microscopio óptico. Escala 0,1 mm.

\section{Conservación}

La conservación común de los insectos fósiles de Rubielos de Mora es en forma de una fina película carbonosa, y así ocurre en los cuatro especímenes aquí estudiados. Los diferentes matices de color que se aprecian en los ejemplares, y que varían de marrón muy claro hasta negro, se corresponden con distintos grados crecientes de esclerotización original, pero también con patrones originales de coloración; por ejemplo, en patas (Lám. I, fig. 6) y antenas, los patrones observados en los fósiles nos indican coloración original en comparación con las formas actuales.

Pese a la presunta delicadeza original de estos hemípteros, los fósiles muestran pocos indicios de desarticulación, como son la pérdida de extremidades y de parte del abdomen en algunos ejemplares y principio de desarticulación del pronoto en MPZ 97/555.

Las medidas tomadas en la Tabla 1 deben ser consideradas teniendo en cuenta que los tres ejemplares más completos se presentan fosilizados en posición dorsoventral pero algo ladeada. Esta circunstancia determina que, por efecto de la compresión diferencial, a un lado y otro del ejemplar, las medidas de las estructuras pares varíen algunas centésimas de milímetro. Este ladeado, pese a una posición dorsoventral de máximo equilibrio en la deposición del ejemplar, es debido a la separación que del cuerpo habría experimentado el rostro, tal como se observa en especímenes actuales cuando se guardan en alcohol.

\section{Descripción}

Cuerpo esbelto con fuertes setas semierectas. La longitud del cuerpo es 5,14 veces la anchura del pronoto (ver medidas de los cuatro ejemplares en la Tabla 1).

Cabeza esférica con ojos grandes. El rostro probablemente alcanzaría el nivel de las metacoxas (Figs. 1.a y 2.b). Antenas con el primer artejo de color muy oscuro, el segundo es basalmente claro y presenta una mancha distal oscura y una pilosidad corta de longitud inferior al diámetro del artejo (no se distinguen en él setas largas). Los restantes artejos carecen de pilosidad aparente. Los siguientes dos artejos son de color oscuro, de la misma tonalidad que la mancha distal del segundo. El segundo artejo es 1,39 veces tan largo como la anchura del pronoto y 2,20 veces tan largo como la anchura de la cabeza. El tercer artejo antenal es 0,68 veces la longitud del segundo y 1,87 veces la del cuarto (Tabla 1).

Pronoto trapezoidal. Collar pronotal presente. Aparente ausencia de callosidades en el lóbulo anterior del pronoto. Escutelo grande, triangular, equilátero y con una ancha banda longitudinal marrón (Lám. I, fig. 5).

Hemiélitros hialinos, manchados de marrón en el extremo distal de la coria y en el ángulo posterior del cúneo (Fig. 3 y Lám. I, figs. 3 y 4), tal como es típico en las especies actuales. Las inserciones de las setas en las regiones esclerotizadas de los hemiélitros están marcadas por una mancha más oscura. El borde costal presenta setas semierectas. La membrana es transparente y posee dos células basales que ocupan la zona de sutura con la parte esclerotizada. Las nerviaciones de las celdillas de la membrana son muy patentes y de color marrón.

Los metafémures presentan pilosidad por toda su superficie, algunas setas aisladas y conservan trazas de coloración original en forma de pequeñas manchas oscuras alargadas -MPZ 97/555 (Fig. 2.b y Lám. I, fig. 6) y MPZ-70RM-. Los fémures de las restantes patas presentan el mismo tipo de pilosidad pero sin rastros de coloración. Las tibias están cubiertas de pilosidad de longitud inferior al diámetro del artejo, sin espinas grandes que sobrepasen el diámetro del artejo. La longitud de la metatibia es 4,75 veces la anchura de la cabeza, 3,01 veces la anchura del pronoto y 4,31 veces la longitud de los metatarsos (Tabla 1). Los tarsos triarticulados tienen escasa pilosidad, quizá perdida, siendo los artejos basales de color claro mientras los distales están oscurecidos en el extremo. En algunos ejemplares los tarsos conservan pequeñas uñas ligeramente curvadas.

El abdomen falta por desarticulación en los ejemplares MPV-70-RM y MPV-2244-RM, no siendo posible la determinación del sexo en ningún espécimen.

\section{Discusión}

Los especímenes presentan los caracteres típicos del género actual Dicyphus. Además, estos caracteres, en

Lámina I. Ejemplares de Dicyphus rubusensis nov. sp. (Hemiptera: Miridae) de Rubielos de Mora. Escalas 1 mm.

1 Ejemplar MPV-566-RM, holotipo.

2 Ejemplar MPZ 97/555, paratipo.

3 Hemiélitro izquierdo del holotipo (MPV-566-RM).

4 Detalle de los hemiélitros del ejemplar MPV-70-RM, paratipo.
5 Detalle de la cabeza y tórax del ejemplar MPZ 97/555, paratipo.

6 Detalle de la coloración moteada en el metafemur derecho del ejemplar anterior (la flecha de la ilustración 2 indica el detalle fotografiado). 
Lámina I
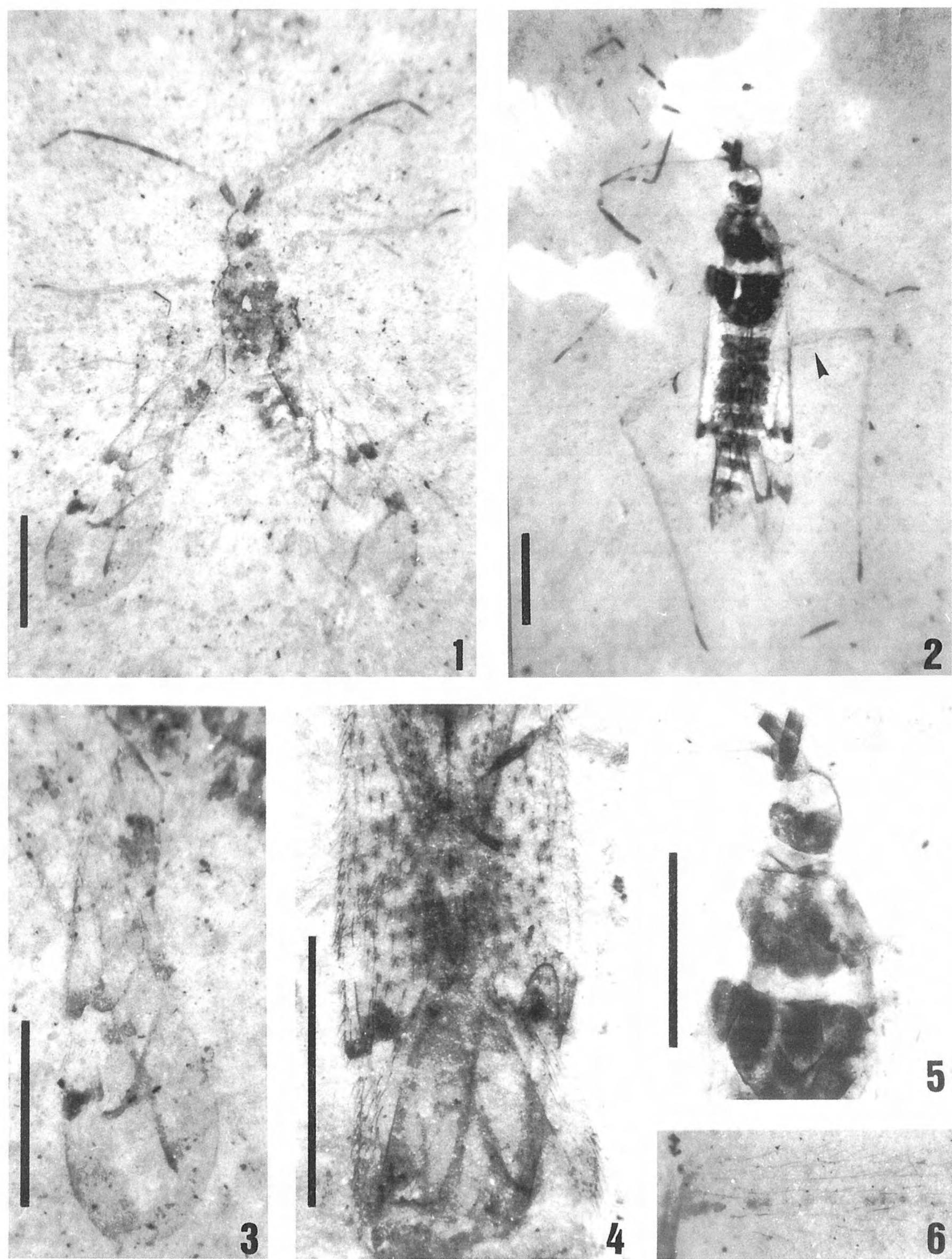

5

Revista Española de Paleontología, 15 (1), 2000 


\begin{tabular}{|c|c|c|c|c|c|}
\hline & $\begin{array}{c}\text { MPV-70-RM } \\
\text { paratipo }\end{array}$ & $\begin{array}{c}\text { MPV-566-RM } \\
\text { holotipo }\end{array}$ & $\begin{array}{c}\text { MPV-2244-RM } \\
\text { paratipo }\end{array}$ & $\begin{array}{c}\text { MPZ 97/555 } \\
\text { paratipo }\end{array}$ & media \\
\hline Longitud corporal & --- & 2,92 & --- & 3,57 & 3,24 \\
\hline Anchura de la cabeza & 0,38 & 0,44 & --- & 0,39 & 0,40 \\
\hline Anchura del ojo & 0,22 & 0,20 & --- & 0,27 & 0,23 \\
\hline Longitud del ojo & 0,27 & 0,25 & --- & 0,20 & 0,24 \\
\hline Longitud del rostro & --- & 1,61 & --- & 1,69 & 1,65 \\
\hline Longitud $1^{\circ}$ artejo antenal & 0,24 & 0,27 & --- & 0,27 & 0,26 \\
\hline Longitud $2^{\circ}$ artejo antenal & 0,83 & 0,85 & --- & 0,97 & 0,88 \\
\hline Longitud $3^{\circ}$ artejo antenal & 0,62 & 0,60 & 0,52 & 0,64 & 0,60 \\
\hline Longitud $4^{\circ}$ artejo antenal & 0,31 & 0,31 & --- & 0,34 & 0,32 \\
\hline Anchura del pronoto & 0,62 & 0,64 & 0,62 & 0,65 & 0,63 \\
\hline Longitud ala anterior & 3,11 & 2,86 & --- & 3,28 & 3,08 \\
\hline Anchura máxima ala anterior & 0,86 & 0,89 & --- & --- & 0,88 \\
\hline Longitud protibia & 1,17 & ---- & $\cdots$ & --- & 1,17 \\
\hline Longitud protarso & 0,41 & 0,36 & --- & --- & 0,39 \\
\hline Longitud mesotibia & 1,07 & 1,17 & 1,14 & 1,28 & 1,17 \\
\hline Longitud mesotarso & 0,38 & 0,34 & --- & 0,34 & 0,35 \\
\hline Longitud metatibia & ---- & 1,80 & 1,67 & 2,22 & 1,90 \\
\hline Longitud tmetaarso & --- & 0,48 & 0,37 & 0,47 & 0,44 \\
\hline Longitud pata metatorácica & ---- & 3,36 & 3,18 & 4,11 & 3,55 \\
\hline
\end{tabular}

Tabla 1. Medidas de los ejemplares de Dicyphus rubusensis nov. sp. (Hemiptera: Miridae), en milímetros. Algunas medidas son la media de las correspondientes a la estructura izquierda y derecha si ambas se han conservado.

general, los acercan al subgénero Dicyphus (Ribes, comunicación oral, 1997). No obstante, el género Dicyphus en la actualidad presenta callosidades en el lóbulo anterior del pronoto, no fosilizadas en los ejemplares estudiados. Dada la conservación que se observa en los cuatro fósiles, la ausencia de las callosidades es dificil de explicar como debida a los procesos de fosilización, por lo que no se puede descartar una ausencia original de estas estructuras en la nueva especie.
D. rubusensis nov. sp., por su biometría y habitus, se puede incluir en el grupo actual hyalinipennis, de cuyas especies se puede diferenciar por la ausencia de setas espinosas de mayor longitud que el diámetro tibial en las meso y metatibias. De las especies del grupo, se asemeja a $D$. cerastii Wagner, 1951, de la que se separa por su menor tamaño y proporciones somáticas.

El ejemplar MPZ 97/555 presenta un tamaño sensiblemente mayor que los otros tres. Ello puede ser debido a dimorfismo sexual, este ejemplar podría 
corresponder a una hembra y el resto a machos, o puede ser debido a un cambio en el tamaño de la especie en el tiempo (no esta determinada la separación microestratigráfica de los diferentes ejemplares y ello puede implicar una separación temporal importante). No obstante, la diferencia de tamaño entra dentro de la variabilidad general de Dicyphus (Goula, comunicación oral, 1998). Aparte de lo anterior, no apreciamos diferencias entre los cuatro ejemplares por lo que los incluimos dentro de Dicyphus rubusensis nov. sp.

\section{BIOGEOGRAFÍA Y BIOLOGÍA DEL GÉNERO Dicyphus}

Respecto a la distribución biogeográfica de los representantes actuales del género Dicyphus, éstos se encuentran principalmente en la región Holártica, con algunos representantes aislados en las regiones Etiópica, Neotropical y Oriental. Respecto a su biología, las diversas especies viven sobre ciertos géneros de plantas pertenecientes a las familias Asteraceae, Boraginaceae, Caprifoliaceae, Caryophylaceae, Chenopodiaceae, Euphorbiaceae, Fabaceae, Geraniaceae, Lamiaceae, Lentibulariaceae, Onagraceae, Pedeliaceae, Rosaceae, Scrophulariaceae, Solanaceae y Urticaceae. Las especies vegetales sobre las que viven comparten una característica común, son plantas pilosas y/o glandulares. El régimen alimentario es fitozoófago, aprovechando en muchos casos los pequeños insectos que quedan atrapados en los pelos y glándulas de las plantas sobre las que viven. Algunas especies actuales han llevado esta adaptación hasta el extremo de cleptoparasitar plantas carnívoras, como Pinguicola, y otras son empleadas por el hombre en la lucha integrada contra ciertas plagas de invernadero.

\section{AGRADECIMIENTOS}

A la Dra. Marta Goula de la Universitat de Barcelona por la lectura crítica del manuscrito y al Dr. Gerry Cassis del Australian Museum de Sydney por la confirmación de la asignación genérica de los ejemplares. Los Drs. Aleksander Herczek, de la Silesian University en Polonia, y Yuri A. Popov, del Intituto de Paleontología de la Academia Rusa de Ciencias en Moscú, revisaron una parte de los ejemplares y nos hicieron interesantes comentarios. Agradecemos al Instituto de Estudios Turolenses la financiación de una excavación paleontológica en septiembre de 1994, en la que fue hallado uno de los ejemplares. Asimismo, agradecemos las facilidades dadas por el Museo de Ciencias Naturales de Valencia y el Museo de Paleontología de la Universidad de Zaragoza para el estudio de los ejemplares. Por último, agradecemos a los dos revisores del manuscrito, los Drs. Conrad C. Labandeira y Xavier Martínez Delclòs, cuyas apreciaciones han mejorado este artículo de manera notable y a la editora Dra. $\mathrm{M}^{\mathrm{a}}$ Luisa Martínez Chacón por su ayuda.

\section{BIBLIOGRAFÍA}

Anadón, P., Cabrera, L., Julià, R., Roca, E. and Rosell, L. 1989. Lacustrine oil-shale basins in Tertiary grabens from NE Spain (Western European rift system). Palaeogeography, Palaeoclimatology, Palaeoecology, 70, 7-28.

Baerensprung, F. 1860. Catalogus Hemipterorum Europaea. Hemiptera Heteroptera Europaea systematice disposita. Berliner Entomologische Zeitschrift, 4 (appendix), 1-25.

Carpenter, F.M. 1992. Superclass Hexapoda. In: Treatise on Invertebrate Paleontology (Ed. R.L. Kaesler). R, Arthropoda, 3, 276 pp.

Fallén, C.F. 1807. Monographia Cimicum Sveciae. C.G. Proft, Hafniae, 123 pp.

Fieber, F.X. 1858. Criterien zur generischen Theilung der Phytocoriden (Capsini. auct.) Wiener Entomologische Monatschrift, 2, 289-327, 329-347, 388.

Gil Collado, J. 1926. Nota sobre algunos insectos fósiles de Ribesalbes (Castellón). Boletín del Instituto Geológico de España, 6, $3^{\text {a }}$ serie, 89-107.

Hahn, C.W. 1833. Die Wanzenartigen Insekten. Getreu nach der Natur abgebildet und beschrieben, 1, 119-236.

Labandeira, C.C. 1994. A compendium of fossil insect families. Milwaukee Public Museum Contributions in Biology and Geology, 88, 71 pp.

Latreille, P.A. 1810. Considérations générales sur l'ordre naturel des animaux composant les classes des Crustaces des Arachnides et des Insectes avec un tableau méthodique de leurs genres, disposés en familles. Schoell, París, 444 pp.

Leston, D., Pendergrast, J.G. and Southwood, T.R.E. 1954. Classification of terrestrial Heteroptera (Geocorisae). Nature, 174, 91-92.

Linnaeus. C. 1758. Systema Naturae per regna triae naturae, secundum classes, ordines, genera, species, cum characteribus, differentiis, synonymis, locis. Editio decima, reformata. i-v, 1-824, L. Salvii, Holmiae.

Martínez-Delclòs, X., Peñalver, E. y Belinchón, M. 1991. Primeras aportaciones al estudio de los insectos del Mioceno de Rubielos de Mora, Teruel (España). Revista Española de Paleontología, $\mathbf{n}^{\circ}$ extra., 125-137.

Montoya, P., Peñalver, E., Ruiz-Sánchez, F.J., Santisteban, C., Alcalá, L., Belinchón, M. y Lacomba, J.I. 1996. Los yacimientos paleontológicos de la cuenca terciaria continental de Rubielos de Mora (Aragón). Revista Española de Paleontología, $\mathbf{n}^{0}$ extra., 215-224.

Peñalver, E. und Seilacher, A. 1995. Rubielos de Mora- Eine untermiozäne Fossil-Lagerstätte. Fossilien, 1995 (4), 211-216.

Popov, Y.A. 1968. True bugs of the Jurassic Karatau fauna (Heteroptera). In: Jurassic insects of Karatau. USSR Academy of Sciences, Section of General Biology, Publ. Hause "Nauka", 99-113. Moscow. (In Russian).

Reuter, O.M. 1883. Hemiptera Gymnocerata Europae. Hémiptères Gymnocérates d'Europe, du bassin de la Méditerranée et de l'Asie Russe. III. Acta Societatis Scientiarum Fennicae, 13, 313-496.

Schuh, R.T. 1995. Plant Bugs of the World (Insecta: 
Heteroptera: Miridae): systematic catalog, distributions, host list and bibliography. New York Entomological Society (Ed.), New York, 1329 pp.

Wagner, E. 1951. Zur Systematik der Gattung Dicyphus
(Hem. Het., Miridae). Societas Scientiarum Fennica, Commentationes Biologicae, 12(6), 1-36.

Wolff, J.F. 1804. Icones Cimicum descriptionibus illustratae. Fasc. 4, J. J. Palm. Erlangae, 127-166.

Manuscrito recibido: 12 de abril, 1999 Manuscrito aceptado: 26 de julio, 1999 